\title{
PROOF RULES DEALING WITH FAIRNESS
}

- Extended Abstract - *

Krzysztof R. Apt

Ernst-Rüdiger Olderog

University of Rotterdam

University of Kiel

Abstract. We provide proof rules allowing to deal with two fairness assumptions in the context of Dijkstra's do-od programs. These proof rules are obtained by considering a translated version of the original program which uses random assignment $x:=$ ? and admits only fair runs. The proof rules use infinite ordinals and deal with the original programs and not their translated versions.

\section{Introduction}

One of the troublesome issues concerning non-deterministic and parallel programs is that of fairness. This assumption states roughly speaking that each possible continuation is scheduled for execution sufficiently often. The meaning of a continuation depends on the language considered. For example, in the case of Dijkstra's guarded commands a possible continuation is a branch guarded by a guard evaluating to true. "Sufficiently often" can be interpreted here in a variety of ways the simplest of them being "eventually".

The aim of this paper is to develop a simple proof theoretic approach to the issue of fairness. This approach was originally suggested in APT \& PLOTKIN [1].

We restrict our attention to Dijkstra's do-od-programs whose components are simple while-programs. Each fairness assumption (we study here two of them) can be incorporated here by providing an appropriate equivalent version of the original program which uses the random assignment $\mathrm{x}:=$ ? (set $\mathrm{x}$ to an arbitrary non-negative integer) for scheduling purposes

Author's addresses: K.R. Apt, Faculty of Economics, University of Rotterdam, P.O. Box 1738, 3000 DR Rotterdam, The Netherlands;

E.-R. Olderog, Institut fur Informatik und Praktische Mathematik, Christian-Albrechts-Universität Kiel, Olshausenstr. 40-60, D-2300 Kiel 1, West Germany.

*) The full version of this paper is available as Bericht Nr. 8104, Institut für Informatik und Praktische Mathematik, University of Kiel, March 1981, and has been submitted for publication. 
and admits only fair computations. By applying to this version of progra Hoare-style proof-rules considered in APT \& PLOTKIN [1] we arrive at proof rules dealing with fairness. It should be stressed that these proc rules deal with the original program - the applied transformations are "absorbed" into the assertions leaving the program in question intact. Using these proof rules total correctness of do-od-programs under the assumption of (weak and strong) fairness can be proved. The proof rules use infinite ordinals.

The use of such infinitistic methods seems to be needed in view of the results of EMERSON \& CLARKE [3] who show that termination under fairness assumption is not first order definable. The results of APT \& PLOTKIN [1] imply soundness and relative completeness of our system for a special type of assertion languages - those which allow the use of the least fixed point operator and ordinals.

\section{Definitions}

We consider programs of the form

$$
\mathrm{s}=\underline{\text { do }} \mathrm{B}_{1} \rightarrow \mathrm{S}_{1}[\ldots] \mathrm{B}_{\mathrm{n}} \rightarrow \mathrm{s}_{\mathrm{n}} \text { od }
$$

where the $B_{i}$ are quantifier-free formulas and the $S_{i}$ are deterministic while-programs. We have a simple model of state in mind, viz. $\sigma: v a r \rightarrow \mathscr{L}$ Var denotes here the set of program variables and $D$ is a domain of an interpretation. Thus the meaning of a subprogram $S_{i}$ is a partially defint mapping $\mathcal{M}\left(\mathrm{S}_{i}\right)$ from states to states. To state the notions of fairness and total correctness properly we employ so-called computation sequences of $S$ defined as follows: For $i \in\{1, \ldots, n\}$ and states $\sigma, \sigma^{\prime}$ we write

$$
\begin{aligned}
& \sigma \stackrel{i}{\longrightarrow} \sigma^{\prime} \text { iff } \models B_{i}(\sigma) \text { and } \mathcal{M}\left(s_{i}\right)(\sigma)=\sigma^{\prime} \text { and } \\
& \sigma \stackrel{i}{\longrightarrow} \text { iff } \models B_{i}(\sigma) \text { and } \mathcal{M}\left(s_{i}\right)(\sigma) \text { is undefined. }
\end{aligned}
$$

Computation sequences of $S$ are now exactly those sequences $\xi$ of states which fall into one of the following cases:

$$
\begin{aligned}
& 1^{\circ} \xi=\sigma_{1} \stackrel{i_{1}}{\longrightarrow} \sigma_{2} \stackrel{i_{2}}{\longrightarrow} \ldots \stackrel{i_{m-1}}{\longrightarrow} \sigma_{m} \\
& \text { where } i_{j} \in\{1, \ldots, n\} \text { and } \models\left(\neg B_{1} \wedge \ldots \wedge \neg B_{n}\right)\left(\sigma_{m}\right) . \\
& \text { Then } \xi \text { is said to properly terminate. } \\
& 2^{\circ} \xi=\sigma_{1} \stackrel{i_{1}}{\longrightarrow} \sigma_{2} \stackrel{i_{2}}{\longrightarrow} \ldots \stackrel{i_{m-1}}{\longrightarrow} \\
& \text { where } i_{j} \in\{1, \ldots, n\} . \\
& \text { Then } \xi \text { is said to fail. }
\end{aligned}
$$




$$
\begin{aligned}
& \quad 3^{\circ} \xi=\sigma_{1} \stackrel{i_{1}}{\longrightarrow} \sigma_{2} \stackrel{i_{2}}{\longrightarrow} \ldots \stackrel{i_{j-1}}{\longrightarrow} \sigma_{j} \stackrel{i_{j}}{\longrightarrow} \ldots \\
& \text { where } i_{j} \in\{1, \ldots, n\} \text { and } \xi \text { is infinite. } \\
& \text { Then } \xi \text { is said to diverge. } \\
& \text { (Note that all finite sequences } \xi \text { of maximal length must fall into the } \\
& \text { cases } 1^{\circ} \text { or } 2^{\circ} \text { because } B_{i}(\sigma) \text { is always defined, } i . e . \models B_{i}(\sigma) \text { or } \\
& \models \neg B_{i}(\sigma) \text { holds.) }
\end{aligned}
$$

A computation sequence $\xi$ of $S$ is said to be weakly fair iff $\xi$ is either finite (i.e. properly terminates or fails) or infinite, i.e. of the form

$$
\xi=\sigma_{1} \stackrel{i_{1}}{\longrightarrow} \sigma_{2} \stackrel{i_{2}}{\longrightarrow} \ldots \quad \sigma_{j} \stackrel{i_{j}}{\longrightarrow} \ldots
$$

with $i_{j} \in\{1, \ldots, n\}$, but then fulfils the following condition

$$
\left.\forall i \in\{1, \ldots, n\} \quad\left(\left(\forall j \in \mathbb{N} \models B_{i}\left(\sigma_{j}\right)\right) \rightarrow\left(\exists j \in \mathbb{N} i_{j}=i\right)\right)^{*}\right)
$$

i.e. if $B_{i}$ is almost always true then the $i-t h$ component is infinitely often chosen. In other words we explicitly disallow infinite sequences $\xi$ with

$$
\exists i \in\{1, \ldots, n\} \quad\left(\forall j \in \mathbb{N} \models B_{i}\left(\sigma_{j}\right) \wedge \forall_{j \in \mathbb{N}}^{\infty} i_{j} \neq i\right) .
$$

A computation sequence $\xi$ of $S$ is said to be strongly fair iff $\xi$ is either finite or infinite, i.e. of the form

$$
\xi=\sigma_{1} \stackrel{i_{1}}{\longrightarrow} \sigma_{2} \stackrel{i_{2}}{\longrightarrow} \ldots \sigma_{j} \stackrel{i_{j}}{\longrightarrow} \ldots
$$

with $i_{j} \in\{1, \ldots, n\}$, but then fulfils the following condition

$$
\forall i \in\{1, \ldots, n\} \quad\left(\left(\exists j \in \mathbb{N} \models B_{i}\left(\sigma_{j}\right)\right) \rightarrow\left(\exists j \in \mathbb{N} i_{j}=i\right)\right)
$$

i.e. if $B_{i}$ is infinitely often true then the $i$-th component is infinitely often chosen. In other words we explicitly disallow infinite sequences $\xi$ with

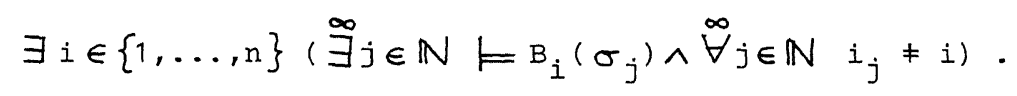

Now we can state precisely what we understand by total correctness of programs with or without fairness assumptions. For arbitrary first order formulas $P$ and $Q$ we define:

*) The quantifier $\stackrel{\infty}{\forall}$ means "for all, but finitely may" and $\stackrel{\infty}{\exists}$ means "there exist infinitely many". 


\section{$\models\{P\} S\{Q\} \quad$ [under weak (strong) fairness assumption]}

iff every [weakly (strongly) fair] computation sequence of $S$ starting in a state $\sigma$ with $\models P(\sigma)$

is properly terminating, i.e. is of the form

$\sigma \stackrel{i_{1}}{\longrightarrow} \ldots \stackrel{i_{m}}{\longrightarrow} \sigma^{\prime}$, and $\sigma^{\prime}$ fulfils $\models Q\left(\sigma^{\prime}\right)$.

Thus under fairness assumption we need not bother about unfair computation sequences.

\section{The Transformations}

Let

$$
s=\text { do } B_{1} \rightarrow s_{1} \square \ldots \square B_{n} \rightarrow s_{n} \text { od. }
$$

We consider the weak fairness assumption first. We use the following transformation

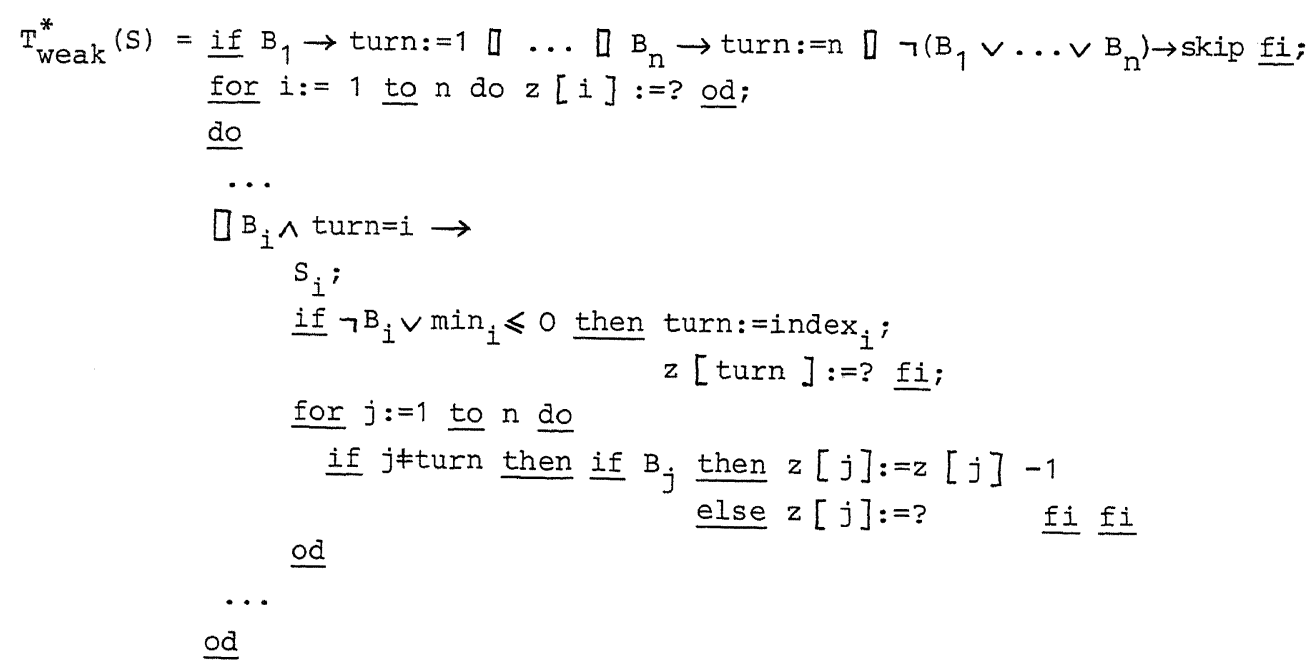

where $i$ ranges from 1 to $n$.

The random assignment $z[i]:=$ ? means "Set $z$ [i] to an arbitrary nonnegative integer". $\min _{i}$ and index $x_{i}$ are shorthands defined as follows

$$
\begin{aligned}
\min _{i} & = \begin{cases}\min \left\{z[j] \mid j \neq i \wedge B_{j}\right\} & \text { if } \underset{j \neq i}{\vee} B_{j} \\
+\infty & \text { otherwise }\end{cases} \\
\text { index }_{i} & = \begin{cases}\min \left\{j \mid j \neq i \wedge B_{j} \wedge z[j]=\min _{i}\right\} & \text { if } \bigvee_{j \neq i} B_{j} \\
i & \text { otherwise }\end{cases}
\end{aligned}
$$


We require that turn and $z[1], \ldots, z[n]$ are variables of $\operatorname{sort}\{1, \ldots, n\}$ resp. integer which do not occur in $\mathrm{S}$.

Some informal explanations may help to understand why $\mathrm{T}_{\text {weak }}^{*}(\mathrm{~S})$ admits only weakly fair runs: If $z[j]>0$ holds, $z[j]$ is equal to the number of times $B_{j}$ is still to be continuously true before control is switched to $S_{j}$ (via turn:=j). Since more than one $z[j]$ can become $O$ at the same time, we allow $z[j]$ to be negative. Each time when $B_{j}$ is true but $S_{j}$ is not executed $z[j]$ gets decreased by 1 . If $B_{j}$ is not true, $z[j]$ is reset to an arbitrary non-negative value. turn=i means that the $i-t h$ component has the control. Control is transferred to another component if $\neg B_{i}$ holds or there exists a $j \neq i$ with $B_{j}$ and $z[j] \leqslant 0$. In both cases control gets transferred to the least component $j$ with the minimal $z$ [j] for which at this moment $B_{j}$ holds. If no such $j$ exists, then all guards $\mathrm{B}_{1}, \ldots, \mathrm{B}_{\mathrm{n}}$ are false and the program terminates.

We now pass to the issue of strong fairness. As basis for our proof rule we take the following transformation

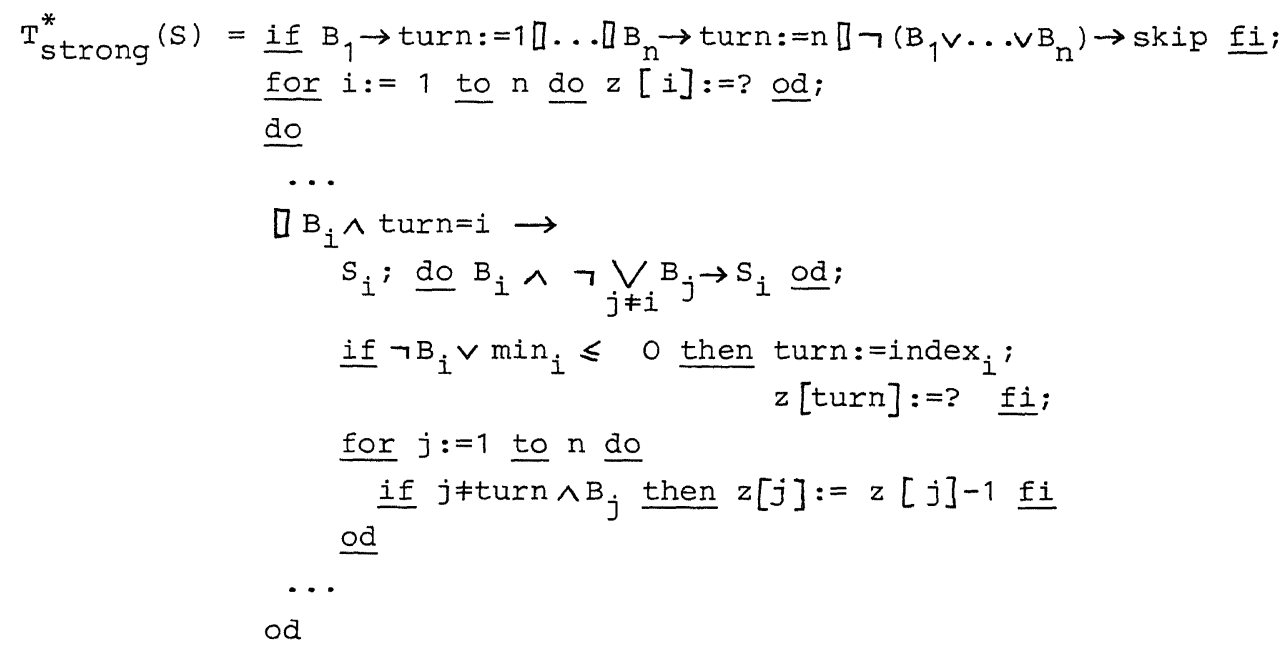


Lemma 1 (a) If $\xi$ is a weakly (strongly) fair computation sequence of $S$ then an element-wise extension $\xi^{\prime}$ of $\xi$ is a computation sequence of $T_{\text {weak }}^{*}(S) \quad\left(T_{\text {strong }}^{*}(S)\right)$.

(b) If $\xi$ ' is a computation sequence of $T_{\text {weak }}^{*}(S)$ ( $T_{\text {strong }}^{*}(S)$ ) then its element-wise restriction $\xi$ to the variables of $S$ is a weakly (strongly) fair computation sequence of $S$.

Intuitively this lemma states that $T_{\text {weak }}^{*}(S)\left(T_{\text {strong }}^{*}(S)\right)$ admits exactly all weakly (strongly) fair computation sequences of $\mathrm{S}$.

\section{The Proof Rules}

The transformed programs use random assignments. In APT \& PLOTKIN [1] proof rules have been developed which allow to prove total correctness of such programs. The relevant proof rule is the following

$$
\begin{aligned}
& \mathrm{P}_{\mathrm{O}} \longrightarrow \exists \propto \mathrm{p}(\alpha) \\
& \mathrm{p}(\mathrm{O}) \rightarrow \neg\left(\mathrm{B}_{1} \vee \ldots \vee \mathrm{B}_{\mathrm{n}}\right) \wedge \mathrm{Q}_{0} \\
& \mathrm{p}(\alpha) \wedge \alpha>0 \rightarrow \mathrm{B}_{1} \vee \ldots \vee \mathrm{B}_{\mathrm{n}} \\
& \frac{\left\{p(\alpha) \wedge \alpha>0 \wedge B_{i}\right\} \quad s_{i} \quad\{\exists \beta<\alpha p(\beta)\} \quad i=1, \ldots, n}{\left\{p_{0}\right\} \quad \text { do } B_{1} \rightarrow s_{1} D \ldots \square B_{n} \rightarrow s_{n} \text { od }\left\{Q_{0}\right\}}
\end{aligned}
$$

where $S_{i}$ are while-programs allowing random assignments and where $\alpha$ and $\beta$ are ordinals.

Below we use the following notation

$$
\begin{gathered}
\bar{z}=(z[1], \ldots, z[n]), \bar{z}=(\underset{\sim}{z}[1], \ldots, \underset{\sim}{z}[n]) \\
\bar{z}-{ }_{i}^{1}=\left(y_{1}, \ldots, y_{n}\right) \\
\text { where } y_{j}= \begin{cases}z[j]-1 & \text { if } j \neq i \text { and } B_{j} \\
z[j] & \text { otherwise }\end{cases} \\
{[\bar{z} \cdot \bar{\sim}]=\left(y_{1}, \ldots, y_{n}\right)} \\
\text { where } y_{j}= \begin{cases}z[j] & \text { if } B_{j} \\
\underset{\sim}{z}[j] & \text { otherwise }\end{cases}
\end{gathered}
$$

Applying the above proof rule to $\mathrm{T}_{\text {weak }}^{*}(\mathrm{~S})$ we derive the following 


$$
\begin{aligned}
& 1^{0} \mathrm{P}_{0} \wedge \underset{i}{\bigvee}\left(\mathrm{B}_{i} \rightarrow \operatorname{turn}=i\right) \rightarrow \forall \bar{z} \geqslant 0 \exists \alpha \mathrm{p}(\alpha, \operatorname{turn}, \bar{z}) \\
& 2^{0} p(0, \operatorname{turn}, \bar{z}) \rightarrow \bigwedge_{i} \neg\left(B_{i} \wedge \operatorname{turn}=i\right) \wedge Q_{0} \\
& 3^{0} p(\alpha, \operatorname{turn}, \bar{z}) \wedge \propto>0 \rightarrow \bigvee_{i}\left(B_{i} \wedge \operatorname{turn}=i\right) \\
& 4 i^{\circ}(i=1, \ldots, n) \\
& \left\{p(\alpha, i, \bar{z}) \wedge \alpha>O \wedge B_{i}\right\} \\
& \left\{\left(\left(\neg B_{i} \vee \min _{i} \leqslant 0\right) \wedge \text { index }_{i}=k \rightarrow\right.\right. \\
& \forall \underset{\sim}{\bar{z}} \geqslant 0 \forall \bar{z}[\mathrm{k}] \geqslant 0 \exists \beta<\alpha \mathrm{p}(\beta, \mathrm{k},[\bar{z}-\mathrm{k} 1 \cdot \bar{\sim}])) \\
& \left(B_{i} \wedge \min _{i}>0 \rightarrow \forall \bar{z} \geqslant 0 p(\alpha, i,[\bar{z}-i 1 \cdot \bar{z}])\right)
\end{aligned}
$$

$\left\{\mathrm{P}_{0}\right\}$ do $\left.\mathrm{B}_{1} \rightarrow \mathrm{S}_{1}\right] \ldots \square \mathrm{B}_{\mathrm{n}} \rightarrow \mathrm{S}_{\mathrm{n}}$ od $\left\{\mathrm{Q}_{0}\right\}$

under weak fairness assumption

where $\alpha, \beta$ are ordinals and turn, $z[1], \ldots, z[\mathrm{n}]$ variables not

occurring freely in $\mathrm{P}_{O^{\prime}} \mathrm{Q}_{\mathrm{O}}$ or $\mathrm{B}_{1}, \ldots, \mathrm{B}_{\mathrm{n}}, \mathrm{S}_{1}, \ldots, \mathrm{S}_{\mathrm{n}}$.

An analogous rule for strong fairness can be derived from $T_{\text {strong }}^{*}(S)$. These proof rules can be considerably simplified in the case of two guards.

Corollary (to Lemma 1). For all formulas $P$ and $Q$ without free variables turn, $z[1], \ldots, z[\mathrm{n}]$ the following holds

$$
\begin{aligned}
& \models\{\mathrm{P}\} \mathrm{S}\{\mathrm{Q}\} \quad \text { under weak fairness assumption } \\
\text { iff } & =\{\mathrm{P}\} \mathrm{T}_{\text {weak }}^{*}(\mathrm{~S})\{\mathrm{Q}\} \quad \text { (under no assumption) }
\end{aligned}
$$

and similarly for the strong fairness assumption.

\section{An Example}

We provide here a natural example for fairness suggested by P. Cousot. Let $I$ be a complete lattice (with ordering $ᄃ$ and least element $\perp$ ) which fulfils the finite chain property, i.e. every strictly increasing $\subseteq$-chain in $L$ is finite. Further on, let $f_{i}: L^{n} \rightarrow L$ be monotone for $i=1, \ldots, n$ and $A, \bar{x}, I, \operatorname{lfp}\left(f_{1}, \ldots, f_{n}\right)$ be the following abbreviations: 
$A=x_{1} \neq f_{1}(\bar{x}) \vee \ldots \vee x_{n} \neq f_{n}(\bar{x})$.

$\bar{x}=\left(x_{1}, \ldots, x_{n}\right)$ and $\bar{I}=(\perp, \ldots, \perp)$ (n times).

$\operatorname{lfp}\left(f_{1}, \ldots, f_{n}\right)$ denotes the least fixed point of the monotone operator $\left(f_{1}, \ldots, f_{n}\right): L^{n} \rightarrow I^{n}$.

Then using our proof rules we can prove

$F\{\bar{x}=\bar{I}\}$

do $A \rightarrow x_{1}:=f_{1}(\bar{x}) \square \ldots \square \quad A \rightarrow x_{n}:=f_{n}(\bar{x})$ od

$\left\{\bar{x}=1 f p\left(f_{1}, \ldots, f_{n}\right)\right\}$

under weak (resp. strong) fairness assumption

where $\models$ refers to validity in $\mathrm{L}$. (This correctness result is a special case of a more general theorem proved in cousot[2].) The full proof can be found in the full version of the paper.

\section{$\underline{\text { References }}$}

[1] APT, K.R. \& G.D. PLOTKIN, A Cook's Tour of countable nondeterminism, Technical Report, Department of Computer Science, University of Edinburgh, 1980 (to appear in Proc. ICALP 81).

[2] Cousot, P., Asynchronous iterative methods for solving a fixed point system of monotone equations in a complete lattice, Rapport de Recherche No 88, L.A.7, Université Scientifique et Medicale de Grenoble, 1977.

[3] EMERSON, E.A. \& E.M. CLARKE, Characterizing correctness properties of parallel programs using fixpoints, in: Proc. ICALP 80, Lecture Notes in Computer Science 85, Springer Verlag, pp. 169-181, 1980. 